EPJ Web of Conferences 33, 02002 (2012)

DOI: $10.1051 /$ epjconf/20123302002

(C) Owned by the authors, published by EDP Sciences, 2012

\title{
Materials for organic photovoltaics: insights from detailed structural models and molecular simulations
}

\author{
Mosè Casalegno ${ }^{1}$, Alberto Baggioli ${ }^{1}$, Antonino Famulari ${ }^{1}$, Stefano V. Meille ${ }^{1}$, Tommaso Nicolini ${ }^{1}$, \\ Riccardo $\mathrm{Po}^{2}$, and Guido Raos ${ }^{1, \mathrm{a}}$. \\ ${ }^{1}$ Dipartimento di Chimica, Materiali e Ing. Chimica “G. Natta”, Politecnico di Milano, via L. \\ Mancinelli 7, 20131 Milano, Italy \\ ${ }^{2}$ Research Center for Non-Conventional Energies, ENI Istituto Donegani, via G. Fauser 4, 28100 \\ Novara, Italy
}

\begin{abstract}
This paper contains a brief discussion of the role of detailed structural and computational studies, within the general field of organic photovoltaics. We review some of our recent work on poly(3-butylthiophene) (P3BT) and on $[6,6]$ phenyl- $\mathrm{C}_{61^{-}}$ butyric acid methyl ester (PCBM). The first is a prototypical hole-transporting material, whose crystal forms I' and II were solved by us through the combined use of powder Xray diffraction, electron diffraction and molecular modelling. PCBM is a widely used fullerene derivative with electron-transporting properties. It has a rich polymorphism, which to date remains largely unexplored. Our molecular dynamics simulations have revealed interesting features of its solid-state organization, including that in the amorphous phase.
\end{abstract}

\section{Introduction}

For a number of reasons - social, economic, environmental — the world is facing the urgent challenge of replacing a significant portion of its fossil fuel consumption by renewable resources.[1] Being virtually inexhaustible, diffuse and extremely abundant, solar radiation is bound to be an important part of the solution. Among the possible strategies for solar energy conversion (see ref. [2] for a broad, up-to-date overview of chemically-oriented research in this field), photovoltaics (PV) is attractive because of the high quality (low entropy content, flexibility of use) of electrical energy. Indeed, there has been a spectacular growth in the global PV industry over the last five years. During 2011 alone, worldwide PV capacity has increased from 40 to 68 GW.[3] Significantly, 60\% this additional power has been installed in Italy and Germany, thanks also to generous government incentives. At the end of 2011, the EU's total installed PV capacity was $47 \mathrm{GW},[3]$ to be compared with an average electricity consumption of $310 \mathrm{GW}[4]$ (of course, the first figure is a theoretical maximum which overestimates the real power output, while the second one is a yearly average which underestimates peak power consumptions).

Today, most of the installed PV power is produced by modules based on $\mathrm{Si}$, which have a theoretical power conversion efficiency (PCE) around 33\% (thermodynamic Shockley-Queisser limit, based on Si's $1.1 \mathrm{eV}$ band gap) and real cell efficiencies around $25 \%$ (monocrystalline $\mathrm{Si}$ ) or

\footnotetext{
a e-mail : guido.raos@polimi.it
} 
20\% (polycristalline Si).[5] Thin film, "second generation" technologies based on amorphous $\mathrm{Si}$, CdTe or CIGS (Copper Indium Gallium Selenide) are also fairly mature and are already present on the market. More advanced PV research mainly addresses "third generation" technologies,[6] which aim at obtaining very high efficiencies (so far up to about $40 \%$, but at much greater cost) or much lower production costs (but with smaller efficiencies). Organic photovoltaic (OPV) cells belong to the latter category, together with other approaches based on nanostructured materials.[7] Lower costs are expected to derive from (a) the use of smaller amounts of cheaper raw materials and (b) the possibility to obtain the OPV cells by fast room-temperature processing methods such as inkjet or roll-to-roll printing, which in addition would allow innovative applications through the use of flexible plastic substrates.[8] The potential of the OPV technology is demonstrated by its certified PCE's, which have increased from about $2 \%$ to $10 \%$ within a decade.[5] Achievement of the $10 \%$ limit is very good news for the OPV field, as this has long been considered a necessary condition for its large-scale deployment. Naturally, there are also other important issues, such as longer cell lifetimes (at least of the order of 4-5 years) and the replacement of ITO (Indium Tin Oxide) as the transparent electrode material.[8]

In the following sections we will briefly present some general ideas and research issues in OPV materials, in order to motivate our work by placing it within a broader context. We will then review our studies of poly(3-butylthiophene) (P3BT) and of [6,6]phenyl- $\mathrm{C}_{61}$-butyric acid methyl ester (PCBM).[9-12] Their molecular structures are displayed in Figure 1, together with a simplified scheme of an organic solar cell (see below). Our aim will be to demonstrate the synergistic roles played by experimental and computational methods in this field.

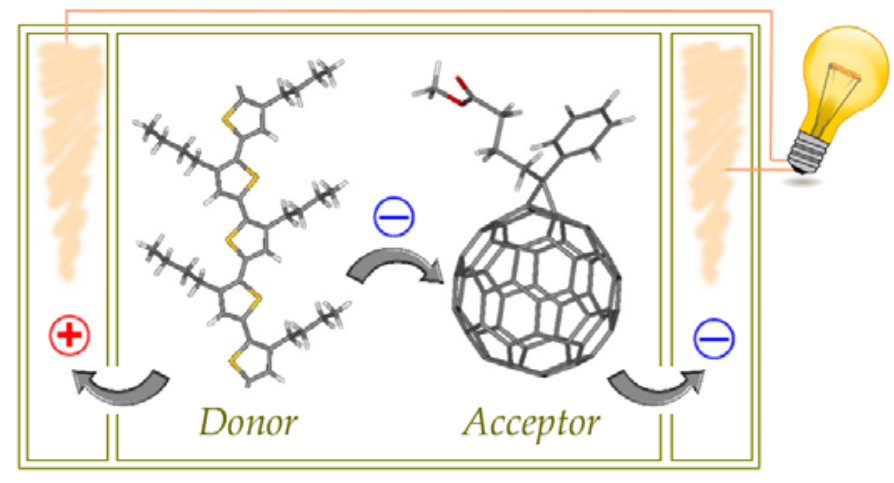

Fig. 1. Scheme of an organic solar cell and molecular structures of regioregular P3BT (left) and PCBM (right). "Regioregular" means that P3BT's monomers are enchained in a regular head-to-tail fashion.

\section{Organic photovoltaic materials}

Most OPV devices are based on the "bulk heterojunction" (BHJ) concept (see ref.[13] for some entry points to a vast and rapidly growing literature). Their active layer is a carefully optimized blend of an electron donor D and an electron acceptor A, which are mainly segregated into two distinct — but percolating and intimately interpenetrated, on the nanoscale — phases. At the interface between an A-type and a D-type material, the excitons generated by light absorption are separated into an electron and a hole, respectively, which are then carried to the cell's cathode and anode to produce an electric current. The OPV materials may be relatively small organic molecules (containing around 100 atoms, say),[14] but more often they contain at least one polymeric component. In any case, they are characterized by a $\pi$-conjugated "skeleton" consisting of $\mathrm{sp}^{2}$ carbons with an alternation of single and double bonds. The relatively weak (compared to the chemical bonds within $\mathrm{Si}$ ) interactions between the molecules of these materials implies significant thermal disorder and strong electron-phonon coupling. As a result, charge transport is usually 
described in terms of discrete electron and hole hopping events, instead of coherent band transport (but see ref.[15] for different opinions on this point). Their charge carrier mobilities are typically in the range $10^{-5}-10^{0} \mathrm{~cm}^{2} /(\mathrm{V} \mathrm{s})$ (the actual values are very sensitive to the degree of structural order, for nominally identical materials[16]), to be compared with $\approx 10^{3} \mathrm{~cm}^{2} /(\mathrm{V}$ s) for inorganic semiconductors. Loosely speaking, the concepts of valence and conduction bands are replaced by those of HOMO (highest occupied molecular orbital) and LUMO (lowest unoccupied molecular orbital) levels. The open-circuit voltage $\left(V_{\mathrm{OC}}\right)$ of an organic solar cell is roughly related to the energy difference between LUMO level of the A material and the HOMO level of the D material.

For some time, the best BHJ system has been the one obtained by blending regioregular poly(3hexylthiophene) (P3HT) with PCBM [13] (P3HT is similar to P3BT, but with six instead of four carbon atoms in the alkyl side chains). The first is a semicrystalline, D-type polymer. The latter is a derivative of the $\mathrm{C}_{60}$ fullerene, with A-type properties. In both cases, the presence of flexible side chains weakens the intermolecular interactions between their $\pi$-conjugated parts and impart them some "room-temperature" $\left(\mathrm{T}<200^{\circ} \mathrm{C}\right)$ processability and solubility in common organic solvents. The BHJ cells obtained from P3HT and PCBM have PCE's of the order of 5\%, after optimization of the deposition method and post-deposition "annealing" treatments.[13] The more recent PCE's of 7$10 \%$ have been obtained by replacing P3HT by some "low gap" copolymers and PCBM by an analogous fullerene derivative, obtained from $\mathrm{C}_{70} \cdot[13(\mathrm{~g})-13(\mathrm{i})]$ These systems were discovered by a combination of some physical/chemical insights (for example, materials with lower HOMO-LUMO gaps collect a larger fraction of the solar spectrum) and a lot of trial-and-error. More recently, extensive quantum chemical calculations have started to be used to identify potentially interesting photovoltaic materials among thousands, possibly even millions, of candidates.[17]

Understanding the structure of BHJ photoactive films and correlating it with their performance has proven to be very challenging, due to the wide range of relevant length scales which contribute to this structure (0.1-100 nm, from intermolecular distances up to the typical device thickness). Incidentally, this problem is shared by many other nanostructured systems.[18] It is however a necessary task, if we want to improve these materials and devices through rational design. Several experimental studies have focused on the PCBM/P3HT blend, which is still a paradigmatic system for fundamental investigations of the structure-morphology-property-efficiency relationships. Many of these have concerned the analysis of morphological aspects (from $1 \mathrm{~nm}$ upwards) by atomic force microscopy (AFM), transmission electron microscopy (TEM) and even three-dimension electron tomography (ET),[19] and their dependence on the previously mentioned thermal or solvent annealing treatments. At the same time, it is generally recognized that some material properties such as the electron and hole mobilities in the PCBM-rich and P3HT-rich domains - depend on more detailed features, such as the structure of the particular polymorph adopted by either material upon crystallization (if it crystallizes at all). $[15,16]$ Our approach has been to examine such detailed structural features, starting from the different phases of pure (unblended) P3BT [9,10] and PCBM.[11] We have done this by combining information from differential scanning calorimetry (DSC), wide-angle X-ray scattering (WAXS), selected-area electron diffraction (SAED) and molecular modeling. The latter includes both ab initio electronic structure calculations and molecular dynamics (MD) simulations.[12]

\section{Structure and modelling of P3BT}

P3BT is the simplest among the poly(3-alkylthiophenes) (P3AT's) of practical interest in organic electronics and photovoltaics. P3HT and the other P3AT's with longer side chains have some obvious similarities to it, leading to analogies in their phase behaviour and solid-state organization. It has been known for a long time that, depending on a number of factors (molecular weight, degree of regioregularity, solvent and deposition conditions, etc.) these may crystallize in two different families of polymorphs, called form I and form II (see ref.[20] for some early work, refs.[9,10] for a more extensive list). These can be easily differentiated from the first peak in their WAXS spectra, which is associated with the distance between $\pi$-stacked layers of chains. In P3BT, this is $\approx 12.5 \AA$ in 
form I $\left(2 \theta \approx 7.0^{\circ}\right.$ with $\mathrm{Cu} \mathrm{K} \alpha$ radiation), which becomes $\approx 9.8 \AA\left(2 \theta \approx 9.1^{\circ}\right)$ in the less common form II. These were often described as "non-interdigitated" and "interdigitated", respectively, as its was believed that interdigitation of the alkyl side chains was necessary to achieve the short interstack distance of form II. However, these interpretations were never supported by detailed structural models (space group, lattice parameters and atomic coordinates). The existence of such detailed models is a necessary conditions for a number of purposes, such as the calculation and rationalization of charge carrier mobilities by molecular modeling methods.[15]

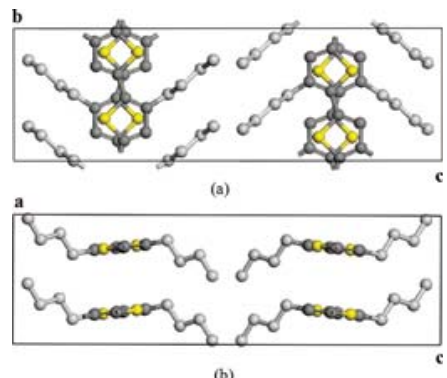

(b)
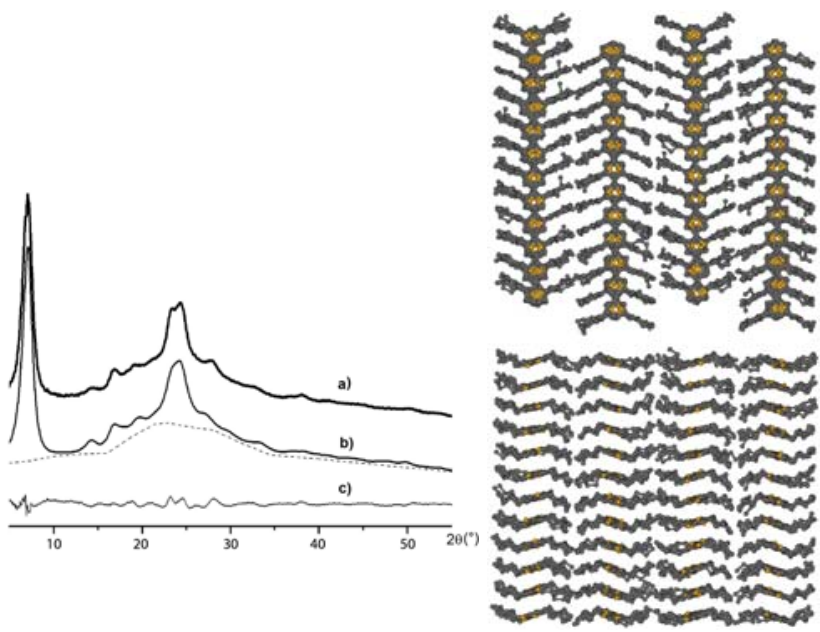

Fig. 2. Results for form I' of P3BT. On the left, we show two views of its $\mathrm{C} 222_{1}$ unit cell (lattice parameters: $a=7.64 \AA, b=7.75 \AA, c=24.97 \AA$ ). In the centre, we show a comparison on the experimental and calculated WAXS profiles. On the right, we show two snapshots from a room-temperature MD simulation of a supercell.

Our experimental work was carried out on highly regioregular, low molar mass P3BT samples. These two conditions allow the formation of highly crystalline samples (note that, in a polymeric material, "high crystallinity" may mean $<50 \%$ of amorphous phase). Indeed, with these samples we found evidence of a new form (called I') for P3BT, which is similar but more ordered than form I.[9] Tentative unit cells were assigned on the basis of the available diffraction data. These were monoclinic or orthorhombic for form I',[9] monoclininc for form II [10] (in the second case, the assignment took account of earlier SAED data by Lu et al.[21], who however had interpreted them in terms of an orthorhombic cell). Preliminary to any further analysis of the experimental data, likely space groups compatible with the unit cells were analyzed in terms of crystallographic symmetry operations and efficiency of intermolecular packing. Note that, in both cases, the polymer chains adopt a 2 helical symmetry, with a planar conjugated backbone and consecutive alkyl side chains pointing out to opposite sides of the backbone plane (see again Figure 1). Having constructed a series of plausible models, these were then refined by Rietveld analysis of the entire powder diffraction profile. In addition to a converged set of lattice parameters and atomic coordinates, this method provides also non-crystallographic, morphological information such as the polymer crystallites' coherence lengths (a measure of their average size). Finally, these structures were subjected to further molecular mechanics and molecular dynamics simulations using our own force field, which had been developed and thoroughly tested on several thiophene oligomers.[12] This allowed us to rule out some candidate structures, whose computed X-ray spectra were compatible with the experimental ones, but which were not viable from an energetic point of view. The final results are given in Figures 2 and 3, respectively for form I' [9] and form II [10]. 


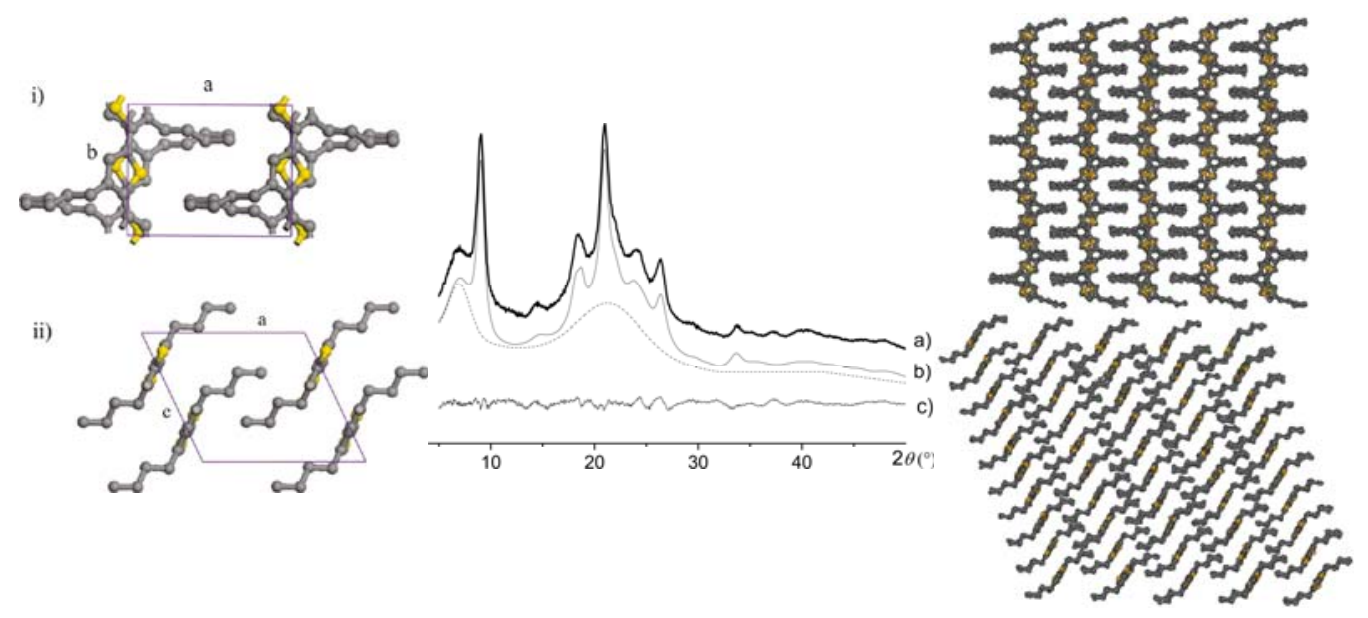

Fig. 3. Results for form II of P3BT. On the left, we show two views of its $\mathrm{P} 2_{1} / \mathrm{c}$ unit cell (lattice parameters: $a=10.76 \AA, b=7.77 \AA, c=9.44 \AA, \beta=64.66^{\circ}$ ). In the centre, we show a comparison of the experimental and calculated WAXS profiles. On the right, we show two snapshot from a room-temperature MD simulation.

The general lessons and insights gained from our work on P3BT may be summarized as follows:

- The unit cell of form I' resembles the previous "inverse comb" model of form I,[20(a)] but with significant additional information. The chains within a stack are iso-directional, those belonging to adjacent stacks anti-directional. The crystal coherence length along the chain axis $(\approx 50 \AA)$, compared with the degree of polymerization of our P3BT samples $(\approx 50$ thiophene units), requires some chain folding (incidentally, the very existence of this folding contrasts with the widespread idea that $\pi$-conjugated polymers are inherently "rigid"). The chain directionalities imply that this folding cannot occur with a stack, but between adjacent stacks. On the other hand, since $\pi$-stacking is likely to be the main driving force for crystallization, it can be expected there will be many intramolecular $\pi$ stacked folds in the pre-crystallization nuclei of high molecular mass chains. This creates a kind of conflict or inhibition, in agreement with the well-known difficulty of crystallization of regioregular P3AT's, especially when they have a high molecular weight.

- The intra- and inter-stack distances of form II are fairly similar, the former being higher and the latter lower than those of forms I and I'. This might suggest a more balanced, threedimensional (as opposed to two-dimensional) charge transport in this form. However it appears more likely that the quality of the tighter stacking is worsened much more than the interstack distances, which remain too high, are improved. Additionally, the very small crystal coherence length along the chain axis $(\approx 25 \AA$, corresponding to ca. 7 thiophene units) points to a high degree of chain folding and plausibly worsens charge mobility along that direction, too. Since crystals are characterized by fast growth in the c direction, the (1 02 ) planes, roughly orthogonal to c, are the more plausible fold planes. In addition, X-ray spectra on oriented thin films show that the $\mathbf{a}$ and $\mathbf{b}$ axes of the crystallites (see Figure 3) are randomly oriented with respect to the substrate, at variance with earlier suggestions of an orthogonal orientation of the chain axes which are parallel to b.[21] Film discontinuity, related to spherulitic morphology, poor stacking and small crystallite dimensions along the chain direction all suggest that crystallization of P3AT's in form II will not be beneficial for OPV applications.

- The MD simulations of the polymers and the oligomers [12] demonstrate that, even in the absence of chain folding, thermal motions can produce significant disordering of the side chains. This is evident in the non-interdigitated form I' (Figure 2), and will certainly be even greater in the more common form I. The side-chain dynamics plays an important role 
also in the mesomorphic phases of these polymers, which may appear at temperatures comparable to those of the typical solar cell annealing treatments.

\section{Solid state organization of PCBM}

Despite of innumerable attempts to replace it by other molecular or polymeric A-type materials,[22] PCBM is still one of the best electron acceptors for solution-processed OPV devices [13]. The factors responsible for its success are not entirely clear, even though they certainly have something do with its high electron affinity and high electron mobility. Significant research efforts have gone in the attempt to clarify the phase behaviour and structural organization of PCBM [23] (see also ref.[24] for a list of structural and morphological studies of P3HT/PCBM blends). These have highlighted a very significant polymorphism. In cast thin films, depending on the solvent evaporation rate, largely amorphous to highly (nano)crystalline samples can be obtained.[23(a)] The SAED data did not allow a solution of the crystal structure in this case. Instead, using extremely slow solvent evaporation conditions, Rispens et al.[25] were able to grow PCBM single crystals suitable for X-ray diffraction. These are in fact co-crystals with the solvent molecules, containing either one ortho-dichlorobenzene molecule per PCBM (in the following, this structure will be called P1D1) or one monochlorobenzene molecule every two PCBM's (this structure will be called P2M1). To date, these are the only two published crystal structures. Little or no solvent molecules are expected to be present in PCBM-based solar cells, after the usual thermal annealing treatments.

Computational methods have been used to develop models for the electronic structure and charge transport in PCBM [26]. However, these simulations were not fully consistent with the available experimental information (in particular, the densities were $10-20 \%$ lower than $1.50 \mathrm{Mg} / \mathrm{m}^{3}$, for amorphous PCBM). In order to assess whether more reliable models could be developed, we performed MD simulations starting from the two co-crystal structures mentioned above, P1D1 and P2M1.[11] These were first simulated "as such", at several temperatures extending up to their apparent melting points (550 and $630 \mathrm{~K}$, respectively). Figure 4 illustrates the results. The good agreement between the calculated and experimental lattice parameters confirmed the reliability of our force field and simulation protocol. Also, the rotational freedom of the solvent molecules within P2M1 (at $400 \mathrm{~K}$, well below the apparent melting point) agreed with their orientational disorder in original crystal structure.[25]

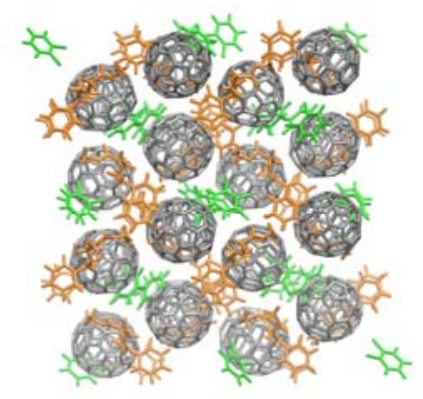

P1D1

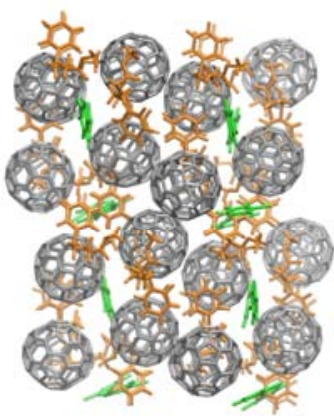

P2M1

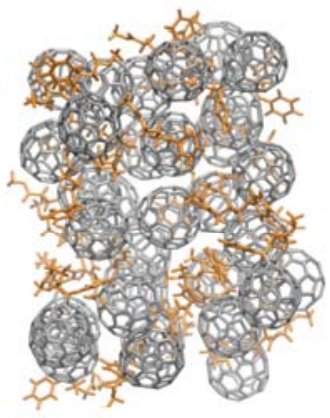

$\mathrm{P} 1 \mathrm{D} 1+\mathrm{M}-\mathrm{S}$

Fig. 4. Snapshots from MD simulations of P1D1 (cocrystal with ortho-dichlorobenzene), P2M1 (cocrystal with monochlorobenzene) and P1D1+M-S (a model for amorphous, solvent-free PCBM). Fullerene portions of

PCBM are shown in grey, side chains in orange, solvent molecules in green.

In order to develop models of solvent-less crystalline PCBM, solvent molecules were removed from P1D1 and P2M1 to obtain structures P1D1-S and P2M1-S. Models for amorphous, solvent-free PCBM were obtained either by melting these structures (P1D1-S+M and P2M1-S+M models) or by first melting the co-crystals and then taking away the solvent molecules (P1D1+M-S and P2M1+M$\mathrm{S}$ models). Table 1 collects our final results (from MD simulations at $400 \mathrm{~K}$ ) on the densities and on the fullerene coordination numbers. The latter were calculated by integrating the fullerene-fullerene pair distribution functions up to an upper cutoff of $1.1 \mathrm{~nm}$. Roughly, they indicate the number of 
neighbouring molecules available for electron hopping. The densities compare well with the available experimental data. The coordination numbers indicate that, even in the amorphous phase, in PCBM there is a well-connected three-dimensional network of conducting path (7 nearest neighbours, which is better than in some crystal structures). This may partly explain the success of this molecule in OPV applications, despite of its noted difficulties of crystallization.

Table 1. Simulated densities and fullerene coordination numbers of the PCBM models, at $400 \mathrm{~K}$.

\begin{tabular}{|c|c|c|}
\hline Model & Density $\left.\mathbf{( M g} / \mathbf{m}^{3}\right)$ & Coord. no. (1.1 nm) \\
\hline P1D1 & 1.625 & 6.00 \\
\hline P1D1-S & 1.523 & 6.62 \\
\hline P1D1-S+M & 1.510 & 6.70 \\
\hline P1D1+M-S & 1.536 & 6.79 \\
\hline P2M & 1.585 & 7.00 \\
\hline P2M1-S & 1.600 & 7.45 \\
\hline P2M1-S+M & 1.529 & 7.01 \\
\hline P2M1+M-S & 1.534 & 7.05 \\
\hline
\end{tabular}

\section{Conclusions}

We have reviewed some of our recent studies of P3BT and PCBM. By doing so, we hope to have demonstrated the usefulness of a combined structural-computational approach to the study of these materials, and the usefulness of the information thus obtained for OPV applications. Further structural and computational work is in progress on PCBM, P3HT and their blends.

Acknowledgments. The authors thank ENI S.p.A. and the Fondazione Cariplo (grant no. 20110349, PLENOS project) for financial support.

\section{References}

1. N. Armaroli and V. Balzani, Energy for a Sustainable World (Wiley-VCH, 2011).

2. A. J. Nozik and A. R. Miller, Chem. Rev. 110, 6443 (2010).

3. European Photovoltaic Industry Association, Market Report 2011. Available at http://www.iepa.org

4. Calculated from a total EU27 electricity consumption of $2.7 \times 10^{9} \mathrm{GWh}$, in the year 2009 . See http://www.iea.org/stats

5. Certified cell efficiencies by the NREL: http://www.nrel.gov/ncpv/

6. M. A. Green, Prog. Photovolt.: Res. Appl. 9, 123 (2001).

7. R. R. Lunt, T. P. Osedach, P. R. Brown, J. A. Rowehl and V. Bulović, Adv. Mater. 23, 5712 (2011).

8. (a) O. Inagäs, Nature Photon. 5, 201 (2011); (b) R. F. Service, Science 332, 293 (2011).

9. P. Arosio, M. Moreno, A. Famulari, G. Raos, M. Catellani, S. V. Meille, Chem. Mater. 21, 78 (2009).

10. A. Buono, N. H. Son, G. Raos, L. Gila, A. Cominetti, M. Catellani, S. V. Meille, Macromol. 43, $6772(2010)$.

11. F. Frigerio, M. Casalegno, C. Carbonera, T. Nicolini, S. V. Meille, G. Raos, J. Mater. Chem. 22, 5434 (2012).

12. M. Moreno, M. Casalegno, G. Raos, S. V. Meille, R. Po, J. Phys. Chem. B 114, 1591 (2010).

13. (a) S. Günes, H. Neugebauer, N. S. Sariciftci, Chem. Rev. 107, 1324 (2007); (b) P. W. M. Blom, V. D. Mihailetchi, L. J. A. Koster, D. E. Markov, Adv. Mater. 19, 1551 (2007); (c) B. C. 
Thompson, J. M. J. Fréchet, Angew. Chem. Int. Ed. 47, 58 (2008); (d) G. Dennler, M. C. Scharber, C. J. Brabec, Adv. Mater. 21, 1323 (2009); (e) B. Kippelen and J. L. Brédas, Energy Environ. Sci. 2, 251 (2009); (f) R. Po, M. Maggini, N. Camaioni, J. Phys. Chem. C 114, 695 (2010); (g) J. Nelson, Mater. Today 14, 462 (2011); (h) G. Li, R. Zhu, Y. Yang, Nature Photon. 6, 153 (2012); (i) H. Zhou, L. Yang, W. You, Macromolecules 45, 607 (2012).

14. A. W. Hains, Z. Liang, M. A. Woodhouse, B. A. Gregg, Chem. Rev. 110, 6689 (2010).

15. (a) V. Coropceanu, J. Cornil, D. A. da Silva Filho, Y. Olivier, R. Silbey, J.-L. Brédas, Chem Rev. 107, 926 (2007); (b) D. L. Cheung, A. Troisi, Phys. Chem. Chem. Phys. 10, 5941 (2008); (c) J. Nelson, J. J. Kwiatowski, J. Kirkpatrick, J. M. Frost, Acc. Chem. Res. 42, 1768 (2009); (d) P. Stallinga, Adv. Mater. 23, 3356 (2011); (e) V. Rühle, A. Lukyanov, F. May, M. Schrader, T. Vehoff, J. Kirkpatrick, B. Baumeier, D. Andrienko, J. Chem. Theory Comput. 7, 335 (2011).

16. A. Selleo, R. J. Kline, D. M. DeLongchamp, M. L. Chabinyc, Adv. Mater. 22, 3812 (2010).

17. (a) N. M. O’Boyle, C. M. Campbell, G. R. Hutchinson, J. Phys. Chem. C 115, 16200 (2011); (b) R. Olivares-Amaya, C. Amador-Bedolla, J. Hachmann, S. Atahan-Evrenk, R. S. SanchezCarrera, L. Vogt, A. Aspuru-Guzik, Energy Environ. Sci. 4, 4849 (2011).

18. S. J. L. Billinge, I. Levin, Science 316, 561 (2007).

19. S. S. van Bavel, J. Loos, Adv. Funct. Mater. 20, 3217 (2010).

20. (a) T. J. Prosa, M. J. Winokur, J. Moulton, P. Smith, A. J. Heeger, Macromolecules 25, 4364 (1992); (b) T. Prosa, M. J. Winokur, R. D. McCullough, Macromolecules 29, 3654 (1996).

21. G. H. Lu, L. G. Li, X. N. Yang, Macromolecules 41, 2062 (2008).

22. (a) F. G. Brunetti, R. Kumar, F. Wudl, J. Mater. Chem. 20, 2934 (2010); (b) Y. He, Y. Li, Phys. Chem. Chem. Phys. 13, 1970 (2011); (c) X. Zhao, X. Zhan, Chem. Soc. Rev. 40, 3728 (2011).

23. (a) X. Yang, J. K. J. van Duren, M. T. Rispens, J. C. Hummelen, R. A. J. Janssen, M. A. J. Michels, J. Loos, Adv. Mater. 16, 802 (2004); (b) L. Li, G. Lu, S. Li, H. Tang, X. Yang, J. Phys. Chem. B 112, 15651 (2008); (c) L. Zheng, Y. Han, J. Phys. Chem. B 116, 1598 (2012); (d) R. Dabirian, X. Feng, L. Ortolani, A. Liscio, V. Morandi, K. Müllen, P. Samorí, V. Palermo, Phys. Chem. Chem. Phys. 12, 4473 (2010).

24. (a) X. Yang, J. Loos, S. C. Veenstra, W. J. H. Verhees, M. M. Wienk, J. M. Kroon, M. A. J. Michels, R. A. J. Janssen, Nano Lett. 5, 579 (2005); (b) J. Y. Kim, C. D. Frisbie, J. Phys. Chem. C 112, 17726 (2008); (c) J. Zhao, A. Swinnen, G. Van Assche, J. Manca, D. Vanderzande, B. Van Mele, J. Phys. Chem. B 113, 1587 (2009); (d) B. Watts, W. J. Belcher, L. Thomsen, H. Ade, P. C. Dastoor, Macromolecules 42, 8392 (2009); (e) R. M. Beal, A. Stavrinadis, J. H. Warner, J. M. Smith, Macromolecules 43, 2343 (2010); (f) N. D. Treat , M. A. Brady , G. Smith, M. F. Toney, E. J. Kramer, C. J. Hawker, M. L. Chabinyc, Adv. Energy Mater. 1, 82 (2011); (g) M. Sanyal, B. Schmidt-Hansberg, M. F. G. Klein, C. Munuera, A. Vorobiev, A. Colsmann, P. Scharfer, U. Lemmer, W. Schabel, H. Dosch, E. Barrena, Macromolecules 44, 3795 (2011); (h) W. Yin, M. Dadmun, ACS Nano 6, 4756 (2011); (i) H.-C. Liao, C.-S. Tsao, T.-H. Lin, C.-M. Chuang, C.-Y. Chen, U-S. Jeng, C.-H. Su, Y.-F. Chen, W.-F. Su, J. Am. Chem. Soc. 133, 13064 (2011); (j) L. Zheng, J. Liu, Y. Ding, Y. Han, J. Phys. Chem. B 115, 8071 (2011); (k) S. Lilliu, T. Agostinelli, E. Pires, M. Hampton, J. Nelson, J. E. Macdonald, Macromolecules 44, 2725 (2011); (1) W. C. Tsoi, S. J. Spencer, L. Yang, A. M. Ballantyne, P. G. Nicholson, A. Turnbull, A. G. Shard, C. E. Murphy, D. D. C. Bradley, J. Nelson, J.-S. Kim, Macromolecules 44, 2944 (2011); (m) P. E. Hopkinson, P. A. Staniec, A. J. Pearson, A. D. F. Dunbar, T. Wang, A. J. Ryan, R. A. L. Jones, D. G. Lidzey, A. M. Donald, Macromolecules 44, 2908 (2011); (n) M. J. Sobkowicz, R. L. Jones, R. J. Kline, D. M. DeLongchamp, Macromolecules 45, 1046 (2012); (o) A. J. Pearson,T. Wang, R. A. L. Jones, D. G. Lidzey, P. A. Staniec, P. E. Hopkinson, A. M. Donald, Macromolecules 45, 1499 (2012).

25. M. T. Rispens, A. Meetsma, R. Rittberger, C. J. Brabec, N. S. Sariciftci and J. C. Hummelen, Chem. Commun. 2116 (2003).

26. (a) J. M. Nápoles-Duarte, M. Reyes-Reyes, J. L. Ricardo-Chavez, R. Garibay-Alonso and R. López-Sandoval, Phys. Rev B 78, 035425 (2008); (b) D. L. Cheung, A. Troisi, J. Phys. Chem. C 114, 20479 (2010). 